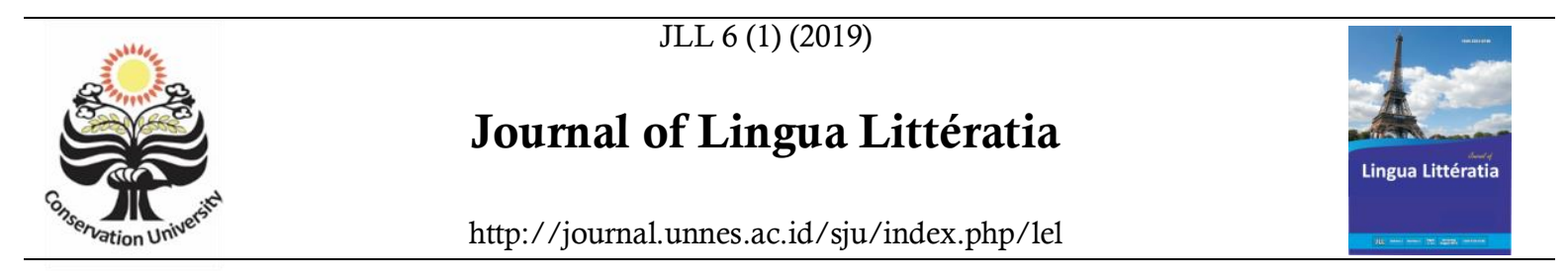

\title{
ANALYSE DE L'ECRANISATION ET L'APPROCHE CULTURELLE DE LA BANDE DESSINEE AU FILM ASTERIX: LE DOMAINE DES DIEUX
}

\author{
Dian Perwitosari $^{\bowtie}$ Sunahrowi \\ Departement de la Langue et la Littérature étrangere, Faculté des Langues et des Arts, \\ Universitas Negeri Semarang, Indonesia
}

\begin{tabular}{|c|c|}
\hline Info d'article & Extrait \\
\hline $\begin{array}{l}\text { Histoire de l'article: } \\
\text { Reçu fevriér } 2019 \\
\text { Accepté avril } 2019 \\
\text { Publié mai } 2019 \\
\text { Mots-clés: } \\
\text { Astérix, Bande Dessinée, } \\
\text { Écranisation, Culture }\end{array}$ & $\begin{array}{l}\text { Astérix : Le Domaine des dieux est le dix-septième album de la série de bande dessinée " Astérix " } \\
\text { de René Goscinny et Albert Uderzo, publié le } 29 \text { juillet 1971. Cette série raconte les aventures du } \\
\text { guerrier gaulois s'opposer aux Romains. Cette bande dessinée a pour fond la région de l'empire } \\
\text { romain vers } 50 \text { av. J.-C. avec l'histoire humoristique. Cette recherche vise à analyser la } \\
\text { transformation dans la bande dessinée au film "Astérix : Le Domaine des dieux » en utilisant la } \\
\text { théorie d'écranisation de Pamusuk Eneste. Cette théorie comprend la réduction, l'addition, et les } \\
\text { changements variés de scène dans le déroulement, le personnage, et le fond. La chercheuse analyse } \\
\text { aussi les éléments culturels en utilisant l'approche culturelle de Koentjaraningrat avec les trois formes } \\
\text { culturelles. Ce sont la forme culturelle comme un complexe des idées, d'activité, et des objets } \\
\text { d'œuvres humaines. Dans cette recherche, la bande dessinée et le film « Astérix : Le Domaine des } \\
\text { dieux » sont choisis comme les objets matériels, tandis que l'objet formel est la théorie d'écranisation } \\
\text { et l'approche culturelle. La chercheuse utilise l'approche qualitative avec la méthode d'analyse } \\
\text { descriptive. La méthode de cette recherche est " la comparaison de référence ", tandis que la } \\
\text { technique de cette recherche est Pilah Unsur Penentu (PUP). Dans l'écranisation du déroulement, il } \\
\text { y a seize réductions, quarante-six additions, et onze variations. En autre, dans l'écranisation du } \\
\text { personnage, il y a deux réductions, cinq additions, et deux variations. Alors, dans l'écranisation du } \\
\text { fond, il y a deux réductions, cinq additions, et deux variations. Dans l'approche culturelle, parmi les } \\
\text { trois formes culturelles que la chercheuse a analysé, on peut voir la troisième forme culturelle est } \\
\text { fidèle aux faits de l'histoire. La troisième forme culturelle de cette bande dessinée décrite les faits } \\
\text { historiques vers } 50 \text { av. J.C. }\end{array}$ \\
\hline
\end{tabular}

(C) 2019 Universitas Negeri Semarang

\begin{tabular}{lc}
\hline Addresse : & ISSN 2252-6730 \\
Gedung B9 Lantai 2 FBS Unnes & \\
Kampus Sekaran, Gunungpati, Semarang, 50229 &
\end{tabular}




\section{L'INTRODUCTION}

La littérature est une œuvre d'art créé par l'auteur ou un groupe de personne en utilisant de la langue. Wellek et Warren (1990 : 109) affirment que la littérature est un lieu pour exprimer des concepts, des idées et des pensées avec des images d'expérience. La littérature est divisée en trois types, ce sont le drame, la poésie et la prose (Isaac et Okunoye $2008: 6$ ).

La prose est divisée en deux types, ce sont la fiction et la non-fiction. La prose fiction est un récit de fiction ou récit d'imagination Nurgiyantoro (2013:2), Stanton dit que la fiction est divisée en deux, à savoir la fiction sérieuse et la fiction populaire (2007: 21-22).

La bande dessinée est l'une fiction qui fait partie de la littérature populaire de Kitsch (Damono $1984: 67-70)$. Elle est une lecture ou une histoire illustrée dans un magazine, un journal ou sous forme de livres (Poerwadarminta $1982: 517$ ).

Dans son livre intitulé Novel dan Film, Eneste (1991 :60) dit que l'écranisation est une transformation d'une œuvre littéraire à un film. Cette transformation se traduira par trois changements afin qu'il puisse dire que l'écranisation est un processus de changement. Les changements qui se produisent dans l'écranisation sont la réduction, l'addition, et les changements variés.

Dans cette recherche, la chercheuse a choisi la bande dessine « Astérix : Le Domaine des dieux » de René Goscinny et Albert Uderzo et le film « Astérix :

Le Domaine des dieux " d'Alexandre Astier et Louis Clichy. Il est basé sur deux raisons : Premièrement, il y a des différences dans le déroulement, le personnage, et le fond entre la bande dessinée et le film. Deuxièmement, il y a les éléments culturels existants dans cette bande dessinée, en particulier les éléments culturels Gallo-Romain vers 50 av. J.-C.

Taylor, comme cité par Prasetiani (2014:29-30), dit que la culture est un complexe qui inclut la connaissance, les croyances, la morale, les lois, les coutumes, etc. Les capacités et les habitudes acquises par les êtres humains en tant que membres de la société.

Basé sur ces raisons au-dessus, les problèmes principaux dans cette recherche sont de décrire l'écranisation du déroulement, du personnage, et du fond entre la bande dessinée et le film « Astérix : Le Domaine des dieux ». Ils comprennent : (1) la réduction ; (2) l'addition ; (3) les changements variés.

Tandis que les problèmes principaux de la culture, Koentjaraningrat

(2015 : 150) divise la forme culturelle en trois formes, à savoir :

- la forme culturelle comme un complexe d'idées, de concepts, de valeurs

- la forme culturelle comme un complexe d'activité

- la forme culturelle comme des objets d'œuvres humaines

\section{LA THEORIE}

\section{Bande dessinée et film}

Dans l'écranisation, le média utilisé est les œuvres littéraires et cinématographiques. Les œuvres littéraires dans cette recherche sont la bande dessinée, sont devenues la référence pour faire le film. Le changement de la bande dessinée au film, changera également le contenu. L'écranisation a un but pour analyser la transformation de langage ou les mots aux medias audiovisuels.

Les œuvres littéraires invitent aux lecteurs pour imaginer de l'histoire librement. Le lecteur a une liberté pour imaginer l'image du personnage, le fond et l'histoire. À l'outre coté, en produisant une œuvre littéraire, l'auteur a provoqué souvent la curiosité du lecteur dans le jeu des mots. Au contraire des œuvres littéraires, le film parle avec des images. Les scénaristes se débattent aux matières plastiques. Il faut qu'ils choisissent le matériel qui peut apporter l'image exacte et clair pour ses films. 
La sélection des matériaux et la détermination du lieu suffisamment donnent une idée de l'histoire de fond (Eneste $1991: 16)$.

\section{Éléments d'œuvres littéraires}

Nurgiyantoro (2013 : 29) dit que les éléments intrinsèques sont les éléments qui construisent la littéraire elle-même. Ces éléments qui forment l'œuvre littéraire. L'élément intrinsèque d'une œuvre littéraire est les éléments qui construisent l'histoire directement. Ces éléments intrinsèques d'une œuvre littéraire deviennent être réalisé. Dans cette recherche, les éléments intrinsèques qui seront analysés sont le changement de la bande dessinée au film surtout le déroulement, le personnage et le fond.

\section{Déroulement}

L'événement est une partie du contenu dans une œuvre littéraire, mais la façon d'arranger l'événement appelé le déroulement. Le déroulement est un élément important de la fiction, car il contient l'histoire d'une œuvre littéraire (Wellek et Warren 1990 : 159).

Nurgiyantoro (2013 : 201) dit que le déroulement ne présente pas souvent une séquence d'événements chronologiquement et de suite, mais une présentation qui peut commencer et se terminer avec n'importe quel événement. Ainsi, la première étape de l'histoire peut être située dans n'importe quelle partie. Théoriquement, le déroulement peut être séquencées ou développées en certaines étapes chronologiquement. Cependant, dans la pratique, il n'est pas toujours soumis à la règle. Théoriquement et chronologiquement, les étapes du

développement du déroulement, à savoir l'étape début, intermédiaire et finale.

\section{Personnage}

Sudjiman, comme cité par Budianta (2008 : 87), dit que le personnage est un individu fictif qui vit de certains événements ou qui se comporte dans des événements de l'histoire. En général, le personnage est des humains, des animaux ou des objets. En plus du personnage principal, il y a d'autres types de personnages, le plus important est le personnage opposé qui est le personnage créé pour compenser le personnage principal.

\section{Fond}

Budianta (2008: 86) dit que le fond est toutes les informations sur le temps, l'espace et l'atmosphère dans événements dans une œuvre littéraire. La description du fond peut être en forme physique, réaliste, documentaire, et aussi une description des sentiments.

Abrams, comme cité par Nurgiyantoro (2013 : 314), le fond est divisé en trois éléments principaux, ce sont l'espace de lieu, l'espace de l'heure et l'espace

de l'environnement social où les événements qui sont racontés. Dans cette recherche, le fond est plus axé à celui de la place, car elle représente tous les aspects de fond.

\section{Écranisation}

La théorie sera utilisée dans cette recherche est l'écranisation de l'œuvre littéraire en film (Eneste 1991 : 60). Cette théorie sera utilisée comme un scalpel montrant le processus de changement de film qui est adapté de la bande dessinée

" Astérix: Le Domaine des dieux ». Boggs comme traduit par Asrul Sani (1992 : 4-13), dit que le film reste quelque chose d'unique, même il a la similarité avec d'autres médias. Les films dépassent le drame parce que le film a la capacité de prendre une variété de points de vue, à partir du mouvement, du temps et de

l'espace illimité. 
Ce processus d'écranisation a provoqué de nombreux changements (Eneste 1991: 61-66) explique que les changements comprennent la réduction, l'addition et les changements variés.

\section{Réduction}

L'écranisation signifie aussi ce qui peut être apprécié pendant des heures ou des jours devrait être changé en ce qui est apprécié ou regardé pendant quatrevingt-dix ou cent minutes. En d'autres termes, les bandes dessinées qui sont épais à des centaines de pages doivent inévitablement couper ou rétrécir quand ils vont être filmés. C'est-à-dire que toutes les choses exprimées dans les œuvres littéraires ne seront pas trouvées dans le film. La réduction peut être trouvée aux éléments-ci : le déroulement, les personnages, les fonds ou d'autres éléments qui existent dans les œuvres littéraires et les films. (Eneste $1991: 61$ ).

\section{Addition}

Puisque les scénaristes et les réalisateurs ont interprété l'œuvre littéraire à filmer, il y a la possibilité d'ajouter ici et là. Par exemple, ajouter à l'histoire, le déroulement, le personnage, le fond ou l'atmosphère. Un réalisateur a certainement une raison de faire cette addition. On peut dire que l'addition est importante du point de vue filmis ou qu'elle est toujours pertinente pour toutes les histoires ou pour diverses autres raisons (Eneste 1991 : 61).

\section{Changements variés}

En plus de la réduction et de l'addition, l'écranisation permet également les variations dans le film. Les œuvres littéraires ne sont pas une excuse ou une raison pour les cinéastes, mais les œuvres littéraires sont vraiment d'être transférées sur un autre media, c'est le film. Puisque les différents outils utilisés, il y a certaines variations ici et là. En outre, dans la projection d'un film a également un temps limité afin que le public ne s'ennuie pas à jouir de la fin, donc pas tout savoir que les problèmes qui existent dans les œuvres littéraires peuvent être déplacés tout au film (Eneste 1991 : 66).

\section{Culture}

La culture est pleine du système des idées, des actions et des chefsd'œuvre dans la vie de la société qui est profité de la société. Ça veut dire presque toute l'action humaine est «culture» parce qu'il y a aussi certains d'actions humaines dans la vie de la société qui ne sont pas de la culture, ce sont des actions instinctives, des réflexes, des actions physiologiques ou des comportements inconsidérés. (Koentjaraningrat $2015:$ 144).

\section{Valeurs, normes, règles}

Koentjaraningrat (2015 : 151) dit que la première forme culturelle est aussi appelée la forme idéale de la culture. La caractéristique de la première forme culturelle est souvent abstraite. C'est abstrait parce que cette forme culturelle ne peut pas être touchée ou photographiée. Sa localisation est dans la tête, dans l'esprit de la société où la culture est vivante. Les anthropologues et les sociologues appellent cultural system ou un système culturel (Koentjaraningrat 2015 : 151).

\section{Actions humaines}

La deuxième forme culturelle s'appelle le système social ou social system, à propos des actions modelées de l'homme lui-même. Ce système social consiste des activités humaines qui s'interagissent, se racontent et se mêlent d'un moment à l'autre, jour après jour, et années après années, toujours selon certains modèles basés sur les coutumes de la conduite. Elle est sous la forme d'une série d'activités humaines qui se passe toujours autour de la vie de la société quotidienne. La caractéristique de cette forme culturelle est souvent concrète ou réelle. 
(Koentjaraningrat $2015: 151)$.

\section{Objets d'œuvres humaines}

La dernière forme culturelle est un objet d'œuvre humaine appelée culture physique. C'est-àdire parce que cette forme culturelle est le résultat physique des activités humaines, des actes et des œuvres dans la société. Cette forme culturelle émerge de la culture. Elle a une caractéristique très concrète parce qu'elle est sous la forme des objets de la création humaine ou des choses qui peuvent être touchées, vues et photographiées. (Koentjaraningrat 2015 : 151).

\section{LA METHODOLOGIE DE LA RECHERCHE}

Le type d'approche qui est utilisé dans cette recherche est l'approche qualitative avec une méthode d'analyse descriptive. La chercheuse utilise la technique de bibliothèque, c'est-à-dire la collecte de données qui fait des analyses sur les livres, les littératures, les enregistrements, les rapports (Nazir 2005 : 111).

La collecte de données dans cette recherche est la méthode de simak (écouter et regarder effectivement). La technique de base est la technique sadap (mettre sur écoute), et ensuite la technique catat (noter) (Sudaryanto 1993 : 133). Pour la technique d'analyse de données, la chercheuse utilise la technique Pilah Unsur Penentu (PUP).

La théorie d'écranisation est ensuite utilisée pour analyser les données. Le corpus utilise est la bande dessinée " Astérix : Le Domaine des dieux » écrit par René Goscinny et Albert Uderzo, et le film réalisé par Alexandre Astier et Louis Clichy.

\section{L'ANALYSE}

\section{Écranisation de la bande dessinée et le film « Astérix: Le Domaine des dieux »}

Dans cette étape, le déroulement, les personnages, et les fonds dans la bande dessinée et le film «Astérix: Le Domaine des dieux » sont apparus dans les séquences. La chercheuse a comparé ces trois éléments dans la bande dessinée et dans le film « Astérix: Le Domaine des dieux » pour trouver la réduction, l'addition, et les changements variés. L'analyse se divise en trois parties : l'écranisation du déroulement, l'écranisation du personnage, et l'écranisation du fond.

\section{Écranisation du déroulement}

Il y a seize données dans la catégorie de la réduction du déroulement.

L'unes de cette partie est comme ci-dessous.
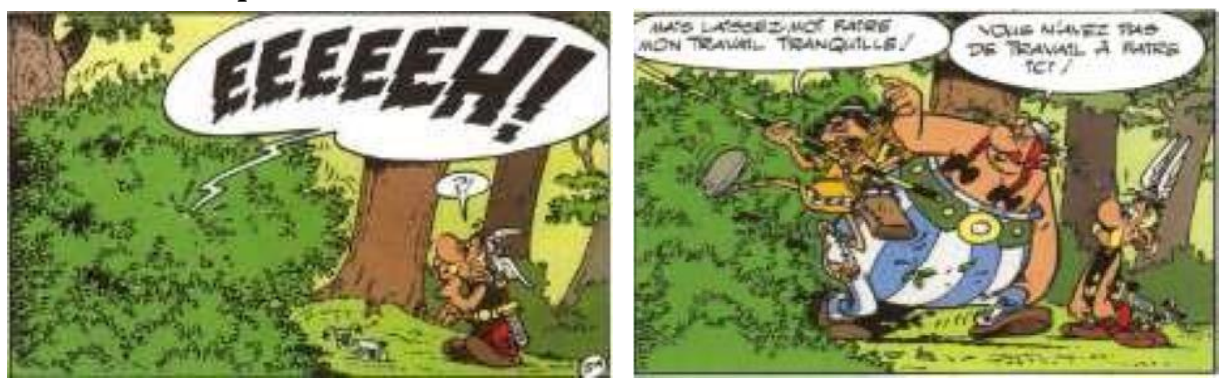

Cette partie se trouve au début de l'histoire. Le réalisateur ne visualise pas cette partie dans la scène du film. Le réalisateur réduit cette partie pour quelques raisons. Cette partie explique qu'Obélix avait élevé Anglaigus qui travaillait dans les buissons. Au début, Obélix entendait les voix des 
buissons. Obélix entre dans les buissons et il a élevé Anglaigus. Anglaigus était en colère contre Obélix, car Obélix avait perturbé son travail. Obélix était mécontent, et puis il a frappé Anglaigus. Cette partie de la bande dessinée ne montre pas dans le film. Le réalisateur ne présente pas cette partie au film.

En outre, il y a quarante-six données dans la catégorie de l'addition du déroulement. L'unes de cette scène est comme ci-dessous.

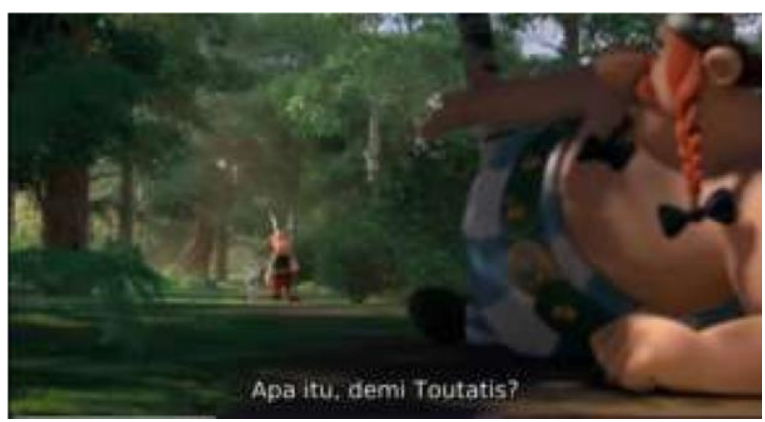

La scène d'Obélix où il est tombé au milieu de la forêt est la première scène supplémentaire qui n'est trouvée pas dans la bande dessinée. Dans cette image, Astérix, Obélix et Idéfix recherchaient des sangliers autour de la forêt. Astérix a trouvé un sanglier, Obélix a également trouvé le même sanglier. Ils se battaient pour le sanglier en courant. Au milieu du voyage, Obélix est tombé. Astérix, qui était derrière Obélix, a vu une corde derrière les buissons. A cause de cette corde, Obélix est tombé.

Par ailleurs, il y a onze données dans la catégorie des changements variés du déroulement. L'unes de cette partie et scène est comme ci-dessous.

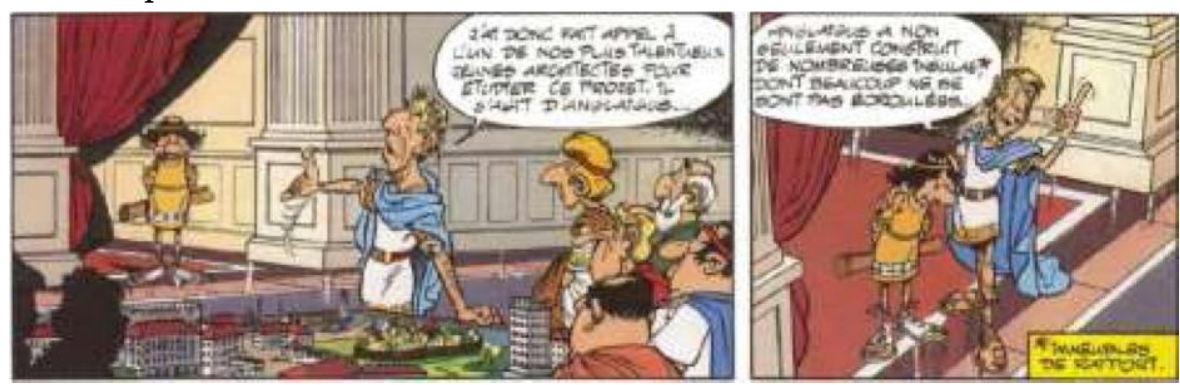

Dans cette partie on sait que Jules César a appelé Anglaigus derrière le rideau rouge. Anglaigus est sorti de derrière le rideau et César s'est approché de lui. Ensuite, César a présenté Anglaigus aux sénateurs.

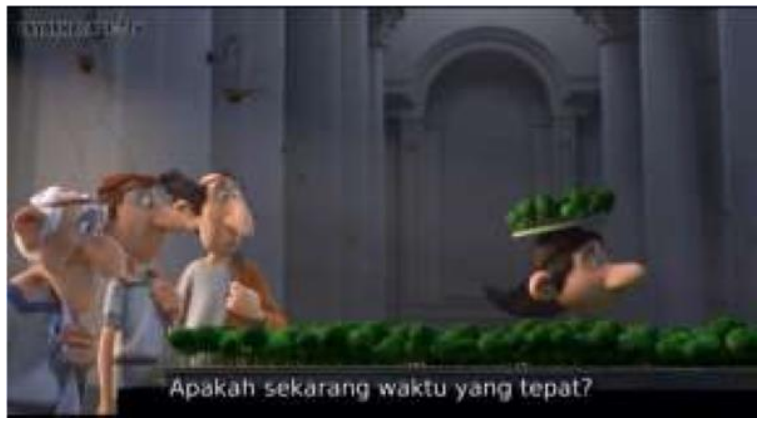

Cette scène est un changement de visualisation qui se trouve dans le film quand Jules César a voulu présenter Anglaigus aux sénateurs. La scène montre Anglaigus qui a apparu sous la table lorsque Jules César a donné un signal. Les sénateurs ont été surpris de voir Anglaigus. César a également présenté l'architecte, Anglaigus, aux sénateurs.

\section{Écranisation du personnage}

Il y a deux donnés dans la catégorie de la réduction du personnage. L'unes de cette partie est comme ci-dessous. 


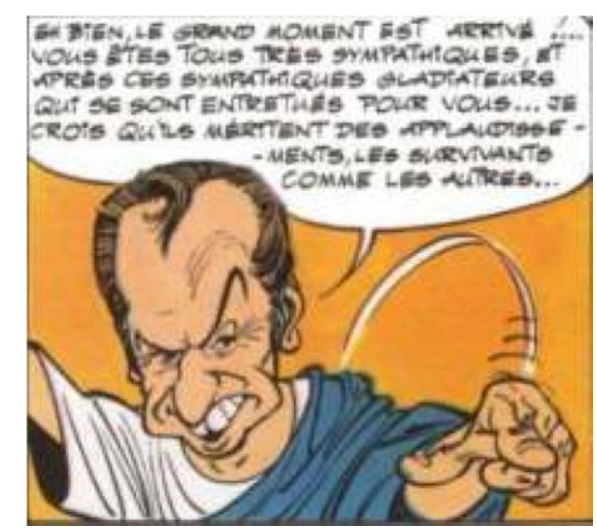

Dans la bande dessinée, Guilus est un sénateur romain qui promeut l'appartement 'Le Domaine des dieux' aux habitants de Rome à l'arène de la lutte. Un autre cas dans le film qui est annoncé 'Le Domaine des dieux' est le sénateur Prospectus. On peut en conclure que le personnage de Guilus est remplacé par un personnage du sénateur Prospectus.

En outre, il y a cinq données dans la catégorie de l'addition du personnage.

L'unes de cette scène est comme ci-dessous.

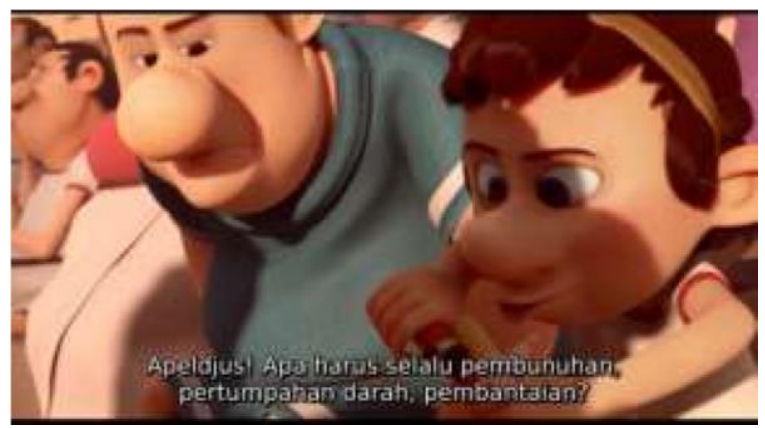

Apeldjus est le fils de Petiminus et de Dulcia. Il a vers 6 à 8 ans. Cette scène dans le film audessus montre que Petiminus a parlé à Apeldus de son jouet.

Dans la bande dessinée, Petiminus et sa femme n'ont pas d'enfants.

Par ailleurs, il y a deux données dans la catégorie des changements variés du personnage. L'unes de cette partie et scène sont comme ci-dessous.

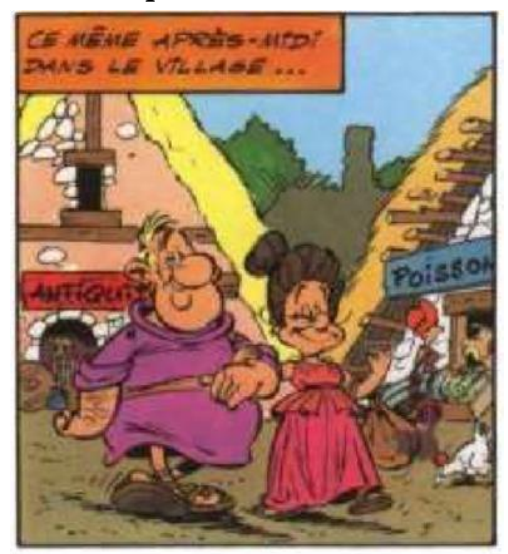

Cette partie montre que l'apparence de Dulcia porte la longue robe rose, et elle n'est pas tellement grande. Son corps est également gras. 


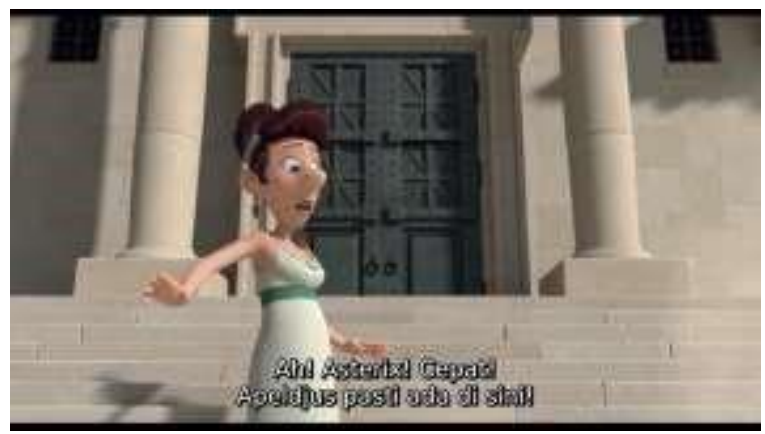

Tandis que dans le film, l'apparence de Dulcia est représentée avec la longue robe blanche et son corps svelte. Elle est également grande. On peut en conclure qu'il y a le changement varié de l'apparence entre la bande dessinée et le film.

\section{Écranisation du fond}

Il y a deux données dans la catégorie de la réduction du fond. L'unes de cette partie est comme ci-dessous.

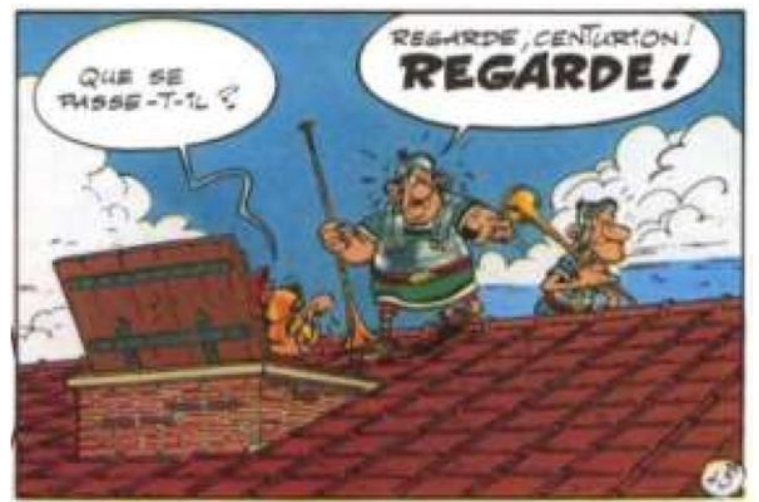

Cette partie montre qu'un soldat romain a dit à Oursenplus que les Gaulois attaquaient l'appartement. Oursenplus se cachait dans le toit de tuiles d'appartement. Tandis que dans le film, Oursenplus ne se cache pas dans le toit de tuiles d'appartement. Cette partie n'existe pas dans le film.

En outre, il y a cinq données dans la catégorie de l'addition du fond.

L'unes de cette scène est comme ci-dessous.

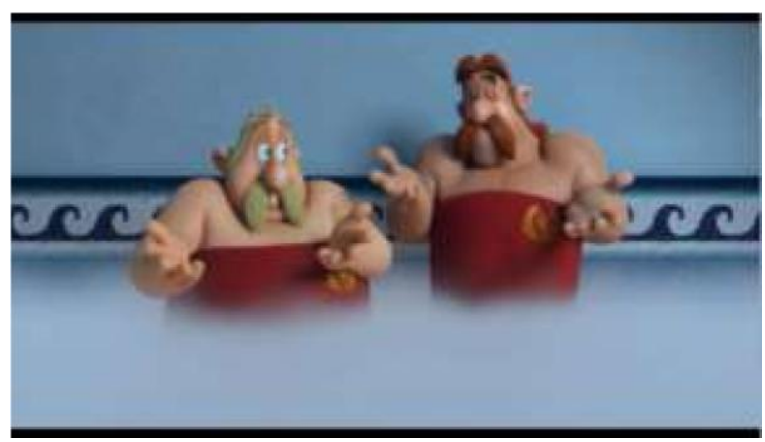

Cette scène prouve qu'il y a l'addition du fond à cause de l'addition du déroulement. Cette scène montre qu'il y a un sauna dans l'appartement 'Le Domaine des dieux', Cétautomatix et Ordralfabétix essayaient d'y être. Tandis que dans la bande dessinée, le fond du sauna n'existe pas.

Par ailleurs, il y a deux données dans la catégorie des changements variés du fond. L'unes de cette partie et scène sont comme ci-dessous. 


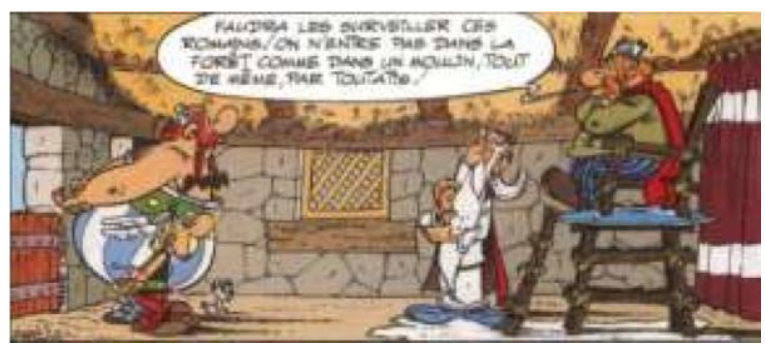

Dans la bande dessinée, Astérix et Obélix ont fait un rapport à

Abraracourcix et à Panoramix dans la hutte d'Abraracourcix. Abraracourcix s'est assis sur une chaise dans sa hutte et Panoramix était à côté de lui.

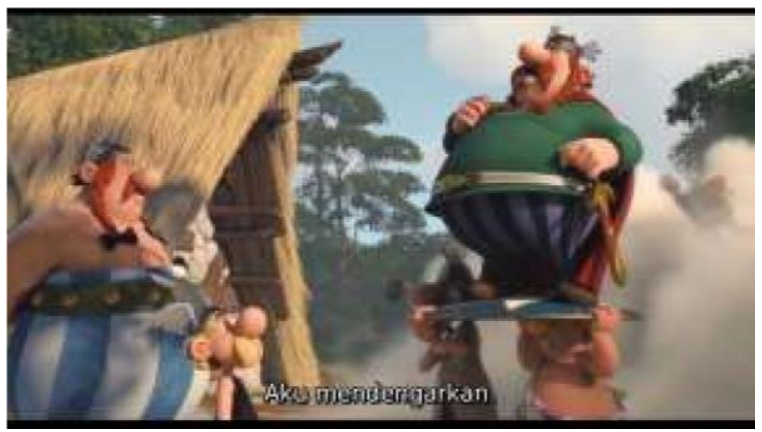

Tandis que dans le film, Astérix et Obélix ont fait un rapport au milieu des Gaulois qui se battaient. Abraracourcix se tenait sur son bouclier qui soulevait par les deux Gaulois. On peut en conclure qu'il y a le changement varié du fond entre la bande dessinée et le film.

\section{Culture de la bande dessinée " Astérix: Le Domaine des dieux "}

Dans cette étape, la chercheuse présente et divise l'unité de l'histoire qui décrit clairement la culture de la Gallo-Romaine. Et pour faciliter la compréhension, après l'exposition des faits historiques contenus dans la bande dessinée "Astérix ", la chercheuse va analyser les éléments culturels de chaque unité de l'histoire par la théorie de Koentjaraningrat.

\section{Contenu de la bande dessinée qui décrit les faits historiques}

Les faits historiques montrent que, jusqu'à 50 av. J.-C., Jules César a tenté d'étendre son territoire à la région de la Gaule. Et même il a réussi conquérir toute la Gaule, cela ne signifie pas que tous les Gaulois sont soumis à ses règles. Les rébellions se passent encore fréquemment à la Gaule (Boak 1961: 225).

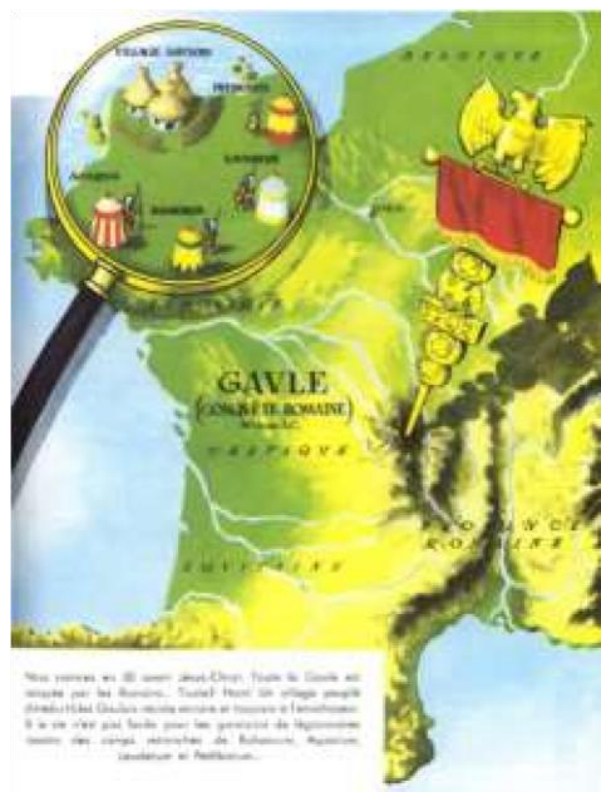


La bande dessinée " Astérix " présente cette histoire de devenir un guide pour les aventures historiques d'Astérix et d'Obélix. Épisode " Astérix: Le Domaine des dieux » fournit d'autres faits historiques à travers la carte qui décrit dans l'image au-dessus. La carte et un peu d'information dans la bande dessinée montrent l'extension de Jules César.

\section{Contenu de la bande dessinée qui décrit la culture gauloise}

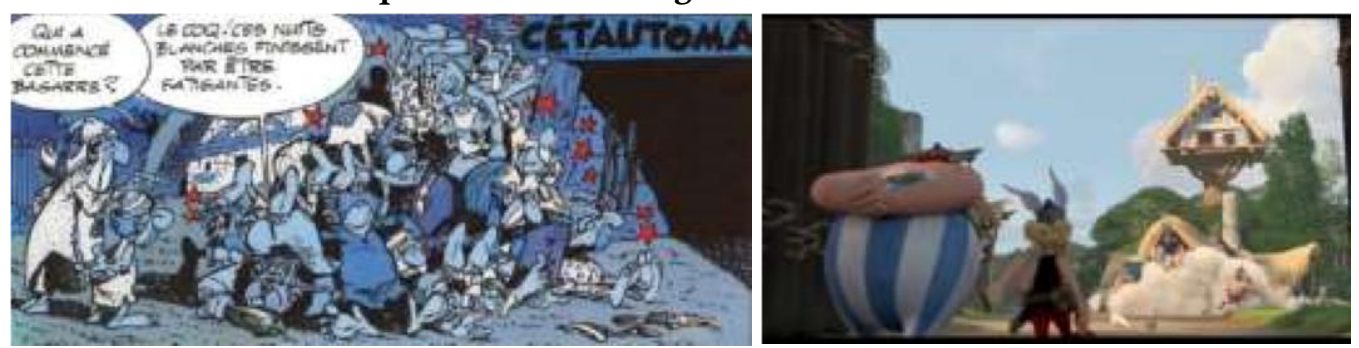

Comme dans la bande dessinée et le film, les Gaulois se disputent toujours. Cette action est l'une des caractéristiques des Celtes qui veulent toujours se battre, avec ses amis et ses ennemis (Haigh 1985: 14).

\section{Contenu de la bande dessinée qui décrit la culture romaine}

La première partie dans la bande dessinée et la première scène dans le film montrent un Aquila. Elles montrent le symbole du pouvoir de César.

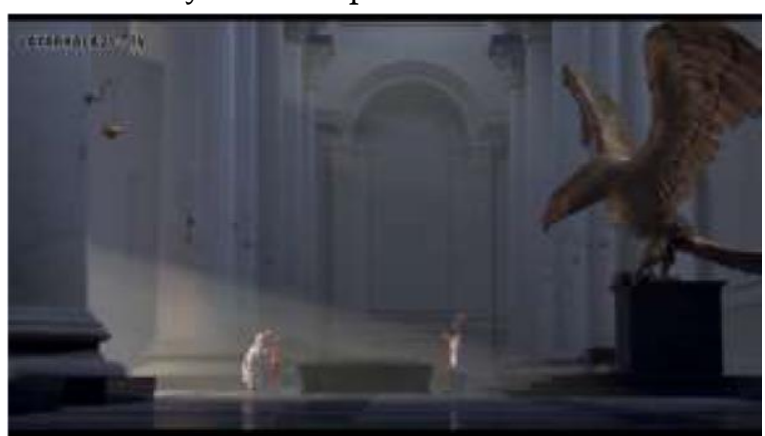

C'est un aigle de bronze ou d'argent est réduit à la taille d'une colombe et monté sur un bâton. Sous l'empire, l'aigle est d'or. Les ailes de l'aigle sont généralement levées, prêtes à mener toute la légion à la victoire, et souvent un éclair en or ou en argent aux serres. L'oiseau est dédié à Jupiter, dieu de la victoire de la légion (Brady 1963 :202).

\section{LA CONCLUSION}

Basée sur la recherche Analyse de l'écranisation et l'approche culturelle de la bande dessinée au film " Astérix : Le Domaine des dieux ", la chercheuse peut tirer quelques conclusions.

Premièrement, après avoir analysé l'écranisation du déroulement, du personnage et du fond de la bande dessinée "Astérix : Le Domaine des dieux » au film, la chercheuse a trouvé plusieurs changements. À l'écranisation du déroulement il y a seize données de la réduction, quarante-six données de l'addition, et onze données des changements variés. À l'écranisation du personnage il y a deux données de la réduction, cinq données de l'addition, et deux données des changements variés. À l'écranisation du fond il y a deux données de la réduction, cinq donnes de l'addition, et il y a deux données des changements variés.

En général, la séquence du déroulement dans la bande dessinée et le film n'a pas beaucoup changé non plus. C'est-à-dire, l'histoire du film n'est pas très loin de celle décrite dans la bande dessinée. Le processus d'écranisation du personnage et du fond, il se produit la réduction et l'addition automatiques parce qu'elles suivent le changement du déroulement dans le film. C'est pareil dans les 
changements variés, ces variations se produisent à cause de certaines raisons du réalisateur. C'est-àdire, le réalisateur veut faire l'histoire plus intéressante.

Après avoir analysé l'adaptation de la bande dessinée au film. La chercheuse a analysé les éléments culturels dans la bande dessinée et le film " Astérix : Le Domaine des dieux », la chercheuse a trouvé les faits historiques à l'époque des Romains envahissent la Gaule vers 50 av. J.-C. Parmi les trois formes culturelles que la chercheuse a analysé, on peut voir la troisième forme culturelle (description de la culture physique) est fidèle aux faits de l'histoire. Les formes physiques de GalloRomaine se présentaient les formes de maisons gauloises avec les appareils ménagers, les vêtements gaulois, les vêtements romains, les uniformes de soldat, etc.

\section{LA BIBLIOGRAPHIE}

Boak, Arthur E.R. 2010. A History of Rome to 565 A. D. New York: The Project Gutenberg EBook.

Boggs, Joseph M. dan Asrul Sani. 1992. The Art of Watching Film (Cara Menilai Sebuah Film). Jakarta: Yayasan Citra.

Brady, Sidney G. 1963. Caesar's Gallic Campaigns. USA : The Stackpole Co.

Budianta, Melani, dkk. 2008. Membaca Sastra: Pengantar Memahami Sastra untu Perguruan Tinggi. Magelang: Indonesia Tera.

Damono, Sapardi Djoko. 1984. Sosiologi Sastra : Sebuah Pengantar Ringkas. Jakarta : Pusat Pembinaan dan Pengembangan Bahasa Departemen Pendidikan dan Kebudayaan.

Eneste, Pamusuk. 1991. Novel dan Film. Flores: Nusa Indah.

Haigh, Christopher. 1985. The Cambridge Historical Encyclopedia of Great Britain and Ireland. Cambridge: Cambridge University Press.

Isaac dan Okunoye. 2008. Literature and Literary. Nigeria : University of Nigeria Press.

Koentjaraningrat. 2015. Pengantar Ilmu Antropologi (Edisi Revisi). Jakarta : Rineka Cipta.

Nazir, Moh. 2005. Metode Penelitian. Jakarta: Ghalia Indonesia.

Nurgiyantoro, Burhan. 2013. Teori Pengkajian Fiksi (Edisi Revisi). Yogyakarta: Gadjah Mada University Press.

Poewardarminta, W.J.S. 1982. Kamus Besar Bahasa Indonesia. Jakarta: Balai Pustaka.

Prasetiani, Dyah. 2014. Aspek Budaya pada Minwa sebagai Identitas Sosial Budaya Masyarakat Jepang (Sebuah Kajian Antropologi Sastra). Jurnal LINGUA Vol.10 No.1.

Sudaryanto. 1993. Metode dan Aneka Teknik Analisis Bahasa: Pengantar Penelitian Wahana Kebudayaan secara Linguistis. Yogyakarta: Duta Wacana University Press.

Wellek, Rene dan Austin Warren. 1990. Teori Kesusastraan (Terjemahan Melani Budianta). Jakarta : Gramedia. 